\title{
THE NEW CHROMOSPHERE
}

\author{
MATS CARLSSON \\ Institute of Theoretical Astrophysics \\ P.O. Box 1029 Blindern, N-0315 Oslo, Norway
}

\author{
AND \\ ROBERT F. STEIN \\ Department of Physics 83 Astronomy, Michigan State University \\ East Lansing, MI 48824, USA
}

\begin{abstract}
The natural state of the Solar chromosphere is very dynamic. Any photospheric disturbance will grow and naturally form shocks over the twenty scale-heights in density between the photosphere and the corona. Observations in the resonance lines from singly ionized calcium and recently in the ultraviolet region of the spectrum observed with the Solar and Heliospheric Observatory satellite also show a very dynamic chromosphere. This dynamic picture is further supported by numerical simulations. Static and dynamic pictures of the chromosphere are fundamentally different. The simulations also show that time variations are crucial for our understanding of the chromosphere itself and for the spectral features formed there.
\end{abstract}

\section{Introduction}

The existence of a million degree corona has been known since Edlén's (1939) identification of coronal emission lines as arising from highly ionized atoms. Initially, the existence of a chromosphere was inferred from the sharp increase in the emission scale height of lines and continua just above the limb of the sun. Starting with the analysis of the 1952 eclipse data by Thomas \& Athay (1961) and others, the chromosphere was inferred from the increase in radiation temperature with increasing opacity in lines and continua. One dimensional models were constructed of the temperature and density that reproduced the observed spatially and temporally averaged continua and line intensities.

Avrett and co-workers (e.g., Vernazza et al. 1981, Maltby et al. 1986, Fontenla et al. 1993) constructed spatially resolved models representing regions of different Lyman continuum intensity corresponding to dark cell interior to very bright network 
locations. These static models show a temperature increasing with height from a minimum value at about $500 \mathrm{~km}$ height above where the optical depth is unity at 500 nm.

Observations with higher spatial and temporal resolution have been made of $\mathrm{CO}$ and Ca II emission (e.g., Solanki et al. 1994, Uitenbroek et al. 1994, and Lites et al. 1993). These results are consistent with a large fraction of the internetwork solar surface having no temperature rise in the first $500 \mathrm{~km}$ above the location of the temperature minimum in the VAL models. The very dynamic behaviour seen in observations of the $\mathrm{Ca}$ II resonance lines even raises the question whether the chromosphere exists as a quasi-static state or if it is wholly dynamic in nature (see e.g., Lites 1985).

The layout of this paper is as follows. In Section 2 we argue that considerations of basic physics leads one to conclude that the natural state of the Solar chromosphere is dynamic and not static, in Section 3 we discuss observations that also imply a very dynamic chromosphere. Section 4 shows that this dynamic picture is also very much supported by numerical simulations. In Section 5 we explain that a static picture and a dynamic picture of the chromosphere are fundamentally different. Section 6 gives some examples of how the temporal variations are crucial for our understanding of spectral features formed in the Solar chromosphere. We conclude in Section 7 with a short summary.

\section{Basic physics}

In the photosphere we have temperatures on the order of $5 \times 10^{3} \mathrm{~K}$ and a gas pressure much larger than the magnetic pressure. In the corona we have temperatures on the order of $10^{6} \mathrm{~K}$, at heights more than $2 \mathrm{Mm}$ above the photosphere, and a magnetic pressure that is much larger than the gas pressure. This corresponds to about 20 scale heights in density and 15 scale heights in gas pressure. The large drop in density and gas pressure has two important consequences:

- perturbations in the photosphere will grow in amplitude with height to conserve wave energy. The growth over 20 scale heights will cause formation of shocks unless we have a very strong damping of the wave.

- while the magnetic field is pushed around by the gas in the photosphere the opposite is true in the corona.

The natural state of the region between the photosphere and the corona is therefore a dynamic state with large amplitude variations. The variations around a mean can be expected to be so large that it is questionable whether a mean atmosphere makes any sense at all. Furthermore, because the magnetic field will start to dominate over the gas somewhere in the chromosphere we expect a one-dimensional nonmagnetic model to break down at some height. A static, one-component model can thus almost be ruled out as a meaningful picture already from very basic physical considerations.

\section{Observations}

It is not altogether straightforward to diagnose the Solar chromosphere from observations. In order for a spectral feature to carry information about the chromosphere the opacity has to be high enough for the radiation to be formed there. In the optical region of the spectrum the continuum opacity is much too low with optical depth unity in the photosphere. We thus need a large line-opacity. Since the opacity is pro- 
portional to the population density of the lower level, this condition translates to lines from the ground state of the dominant ionization stage of an abundant element. Unfortunately, most such lines are found in the ultraviolet part of the spectrum with the notable exception of the resonance lines from singly ionized calcium, Ca II.

These two lines, named the $\mathrm{H}$ and $\mathrm{K}$ lines by Fraunhofer, constitute almost the only available diagnostic of the Solar chromosphere in the optical part of the spectrum and they have therefore received a lot of attention: (e.g., Rutten and Uitenbroek 1991 and references therein; Harvey et al. 1992; Lites et al. 1993; Bocchialini et al. 1994; Von Uexkuell and Kneer 1995; Hofmann et al. 1996; Steffens et al. 1996).

In regions void of strong photospheric magnetic fields (called the inter-network) the $\mathrm{Ca}$ II resonance lines are strongly assymetric and vary in time. A brightening in the line-wing propagates in time towards line-center and ends up as a strong brightening blue-ward of line-center. There is no corresponding brightening on the red side. The line-center absorption is at the same time shifted slowly more and more to the red. Just after the brightening the line-center absorption is abruptly shifted to the blue. In a spectro-heliogram the brightenings of the violet peak can be seen as roundish areas a few megameters wide. They are called $\mathrm{Ca} I I \mathrm{~K}_{2 V}$ (and $\mathrm{H}_{2 V}$ ) bright grains. A brightening in one place often repeats three-four times with about 3 minutes period.

The interpretation of the observations is both simple and complicated. The complication comes from the fact that the line-formation is optically thick and Local Thermodynamic Equilibrium (LTE) is a bad approximation. The good news is that the opacity in the line is a simple function. Almost all calcium is singly ionized in the Solar chromosphere so the opacity is set by the density which is basically given by the hydrostatic stratification. The excitation is collisionally dominated up to rather large heights so the source function does not decouple completely from the Planck function until about $1 \mathrm{Mm}$ above the photosphere. We can thus expect the lines to give information about local conditions in the chromosphere even though modelling is necessary for a detailed understanding. We will return to the interpretation of the observations in Section 4 but it is important to note that even without sophisticated modelling we can conclude from the observations alone that the chromosphere must be very dynamic.

If we also include the ultraviolet part of the spectrum we get access to many more spectral features formed in the chromosphere. All continua shortward of the silicon edge at $152 \mathrm{~nm}$ are formed at heights above $500 \mathrm{~km}$. Variations in the continuum intensity at various wavelengths will give information on the variations in temperature in the chromosphere. Unfortunately, there is no simple inversion to get the physical conditions directly from the observations. The reason is that the source function has decoupled from the Planck function so the intensity is not a good measure of the local temperature. The ionization/recombination may also be out of equilibrium from what the instantaneous values of temperature, density and electron density would imply because of slow recombination rates.

Observations from the SUMER spectrograph on-board the Solar and Heliospheric Observatory (SOHO) satellite show that, indeed, the UV continuum intensities do vary enormously with time (e.g., Carlsson et al. 1997).

\section{Simulations}

To properly model the dynamic Solar chromosphere one has to take into account the important physical processes. Radiative losses are important in continua and lines 
of hydrogen and calcium. The population densities can not be calculated from LTE but has to be solved for using the full rate equations including time-derivative and advection terms because of the slow recombination rates. Shock formation also has to be allowed.

Such a self-consistent radiation-hydrodynamic modelling of the solar chromosphere was performed by Carlsson \& Stein $(1992,1994,1995,1997)$ and we will here summarize some of the results.

A schematic representation of the computational scheme is shown in Fig. 1. We solve the one-dimensional equations of mass, momentum, energy and charge conservation together with the non-LTE radiative transfer and population rate equations, implicitly on an adaptive mesh. We employ 6 level model atoms for hydrogen and singly ionized calcium. We include in detail all transitions between these levels, thus including four Lyman lines $(\alpha, \beta, \gamma, \delta)$, three Balmer lines $(\alpha, \beta, \gamma)$, Paschen $\alpha, \beta$, Bracket $\alpha$, the Lyman-, Balmer-, Paschen-, Bracket- and Pfundt continua of hydrogen, the $\mathrm{H}$ and $\mathrm{K}$ resonance lines, the infrared triplet and the photoionization continua from the five lowest levels of singly ionized calcium. Other continua are treated as background continua in LTE, using the Uppsala atmospheres program (Gustafsson 1973). Microturbulence broadening was set to a constant $2 \mathrm{~km} / \mathrm{s}$ throughout the atmosphere.

Our initial atmosphere is in radiative equilibrium above the convection zone (for the processes we consider) without line blanketing and extends $100 \mathrm{~km}$ into the convection zone, with a time constant divergence of the convective energy flux (on a column mass scale) calculated with the Uppsala code without line blanketing.

Waves are driven through the atmosphere by a piston located at the bottom of the computational domain $\left(100 \mathrm{~km}\right.$ below $\left.\tau_{500}=1\right)$. The piston velocity is chosen to reproduce a 3750 second sequence of Doppler-shift observations in an Fe I line at $\lambda 396.68 \mathrm{~nm}$ in the wing of the Ca H-line (Lites et al. 1993).

The velocity spectrum as a function of frequency changes with height, both in amplitude and phase. The velocity amplitude of propagating waves increases with height in a stratified atmosphere to maintain a constant flux as the density decreases. Damping reduces this amplitude increase. Propagating modes also show a phase shift due to their finite phase speed. Evanescent modes are attenuated but have no change in phase as a function of height.

We calculate this change in amplitude and phase between the piston height and $260 \mathrm{~km}$ as a function of wave frequency and multiply the observed Doppler-shifts with the inverse of this transfer function to obtain our piston velocities. Comparing the simulated velocities at $260 \mathrm{~km}$ with the observed Doppler-shift in the iron line provides a check of this procedure (Fig.1). We applied this procedure to the observed Doppler-shifts at five different slit positions. At the top of the computational domain there is a transmitting boundary condition.

In the dynamic calculation only hydrogen and calcium are treated self-consistently in non-LTE. With the time-variation of hydrodynamic variables (density, temperature, electron density, velocity) taken from this calculation, the statistical equilibrium equations were solved for an additional set of "minority species" (solution assumed not to influence the hydrodynamics or energetics of the atmosphere): neutral aluminium, magnesium, silicon and carbon.

Figure 2 shows that waves are continually traversing the chromosphere. The waves increase in amplitude and form shocks above $1 \mathrm{Mm}$ height. A shock traveling in the cool downflow of a preceding shock may be overtaken by the next shock forming a 


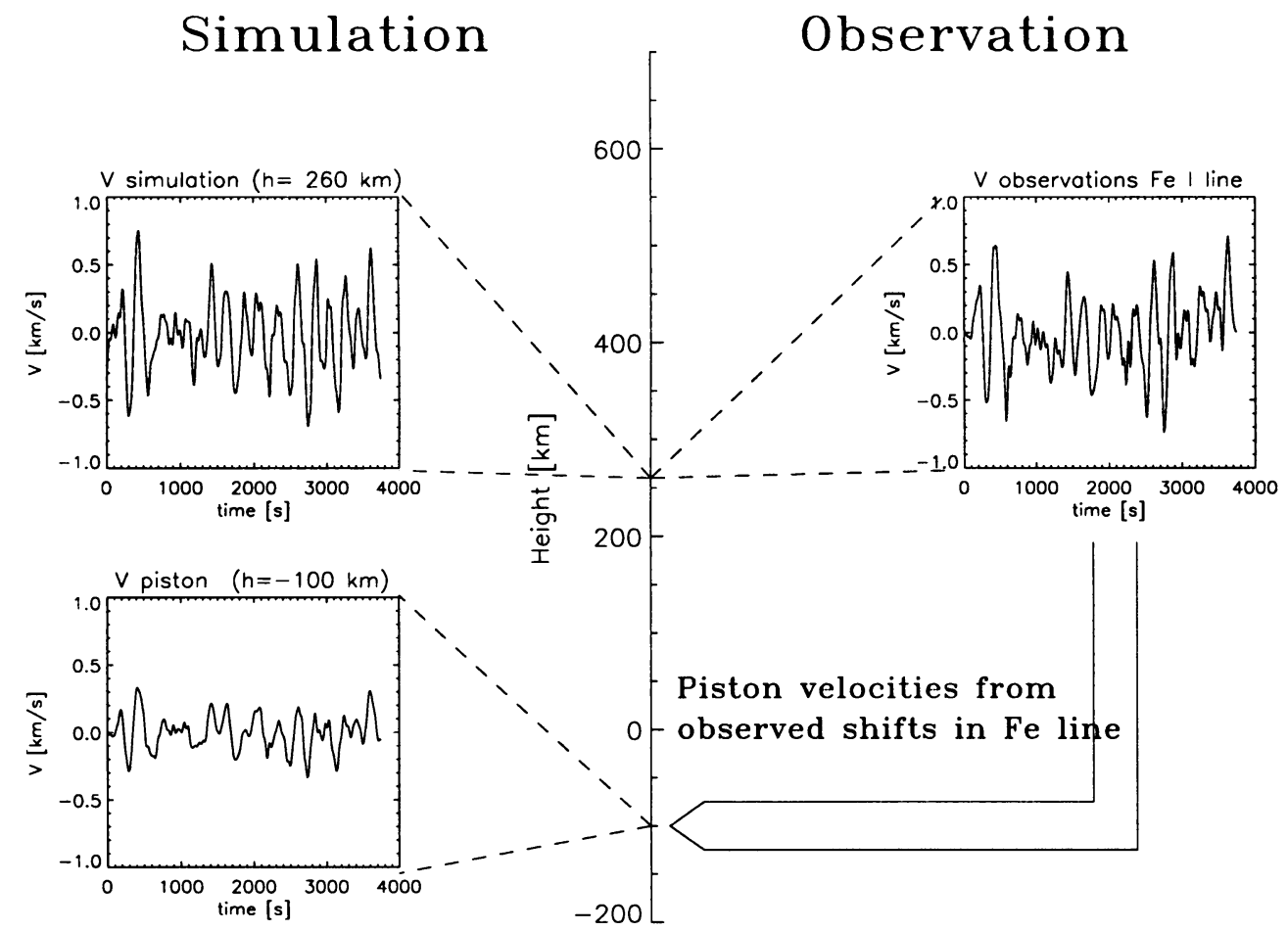

Figure 1. A schematic representation of the computational method. Observed Doppler shifts in an iron line are transformed in amplitude and phase to get a piston velocity that gives a simulated velocity at the iron line formation height $(260 \mathrm{~km})$ close to the observed Doppler-shifts. The equations of conservation of mass, momentum, energy and charge are solved together with the non-LTE rate equations for 6 levels of hydrogen and 6 levels of ionized calcium implicitly on an adaptive mesh.

merged, high-amplitude shock. The temperature difference across a shock can be more than $10^{4} \mathrm{~K}$.

The simulations thus also show that the Solar chromosphere is very dynamic. What can we learn from these simulations? We present one example of how static models can be very misleading and several examples of dynamic diagnostics.

\section{Failure of Static models in a Dynamic Chromosphere}

Classical static models of the Solar chromosphere are based on temporal and spatial averages of intensity - either continuum intensities shortward of the silicon edge at $152 \mathrm{~nm}$ (like in the models by Avrett and co-workers, e.g., Vernazza et al. 1981, Maltby et al. 1986, Fontenla et al. 1993) or line profiles of resonance lines from ionized calcium and magnesium. The crucial factor here is that the mean is taken in the ultraviolet part of the spectrum where the temperature dependence of the Planck function is more exponential than linear. The source function is quite decoupled from 
194 Slit 110

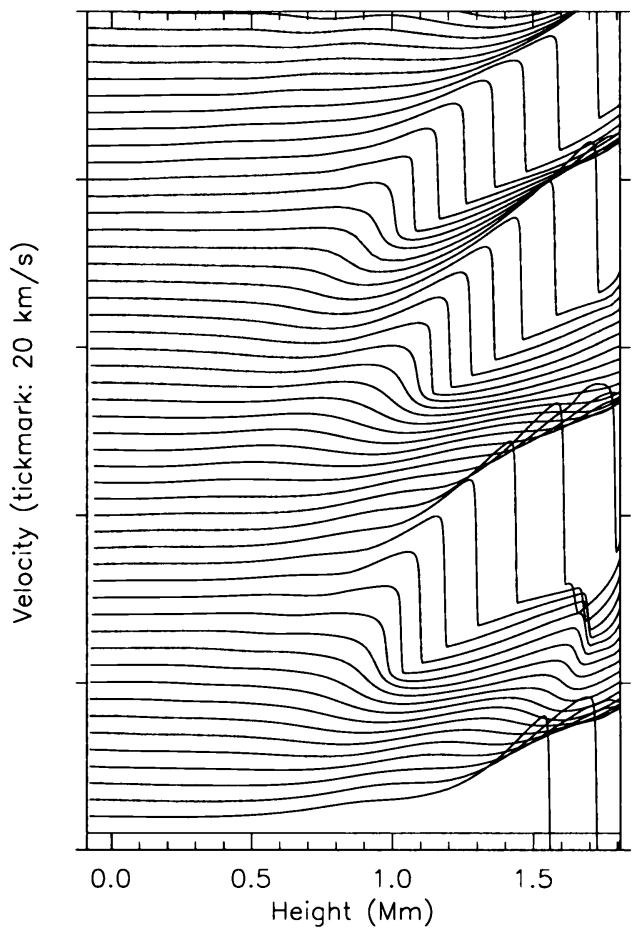

194 Slit 110

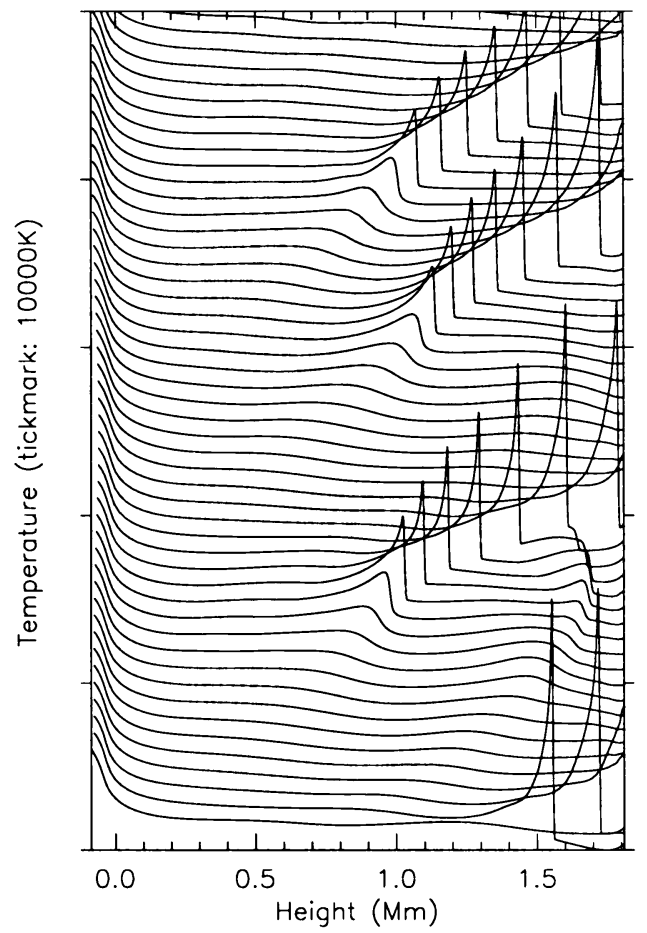

Figure 2. The velocity and temperature as functions of height and time. The curves are 10s apart in time and offset in the vertical direction. The amplitudes can be seen from the tickmarks that are $20 \mathrm{~km} / \mathrm{s}$ apart in velocity and $10^{4} \mathrm{~K}$ apart in temperature. Waves propagate and form shocks above $1 \mathrm{Mm}$ height. Shocks may overtake an earlier shock and merge.

the Planck function and has a smaller amplitude than the local Planck function but the fact remains that a mean preferentially samples the high temperatures. This was dramatically illustrated in Carlsson \& Stein (1995) where the best semi-empirical fit to the mean intensities in the simulation showed a chromospheric temperature rise while the mean temperature showed no increase, see Fig. 3.

This result does not mean that there is no chromosphere. The simulations have enhanced chromospheric emission much above the radiative equilibrium values and not too different from the observed values (see also Section 6.2). This emission is, however, not caused by an increase in the mean temperature - the mechanical work goes directly into radiation instead of first causing a temperature increase.

The effects of waves with frequencies above $20 \mathrm{mHz}$ and of absorption in the helium continuum of radiation from the corona have been neglected in the simulations. Both of these effects would favour a mean temperature rise. The Solar chromosphere may thus well have a mean temperature rise but the essential conclusions remain:

- a mean temperature structure can not be deduced from mean intensities in the blue part of the spectrum. 
- in a dynamic chromosphere the mean temperature is not only difficult to deduce but is also a meaningless and even misleading quantity. The energy balance can only be deduced from a dynamical model and not from any such mean model.

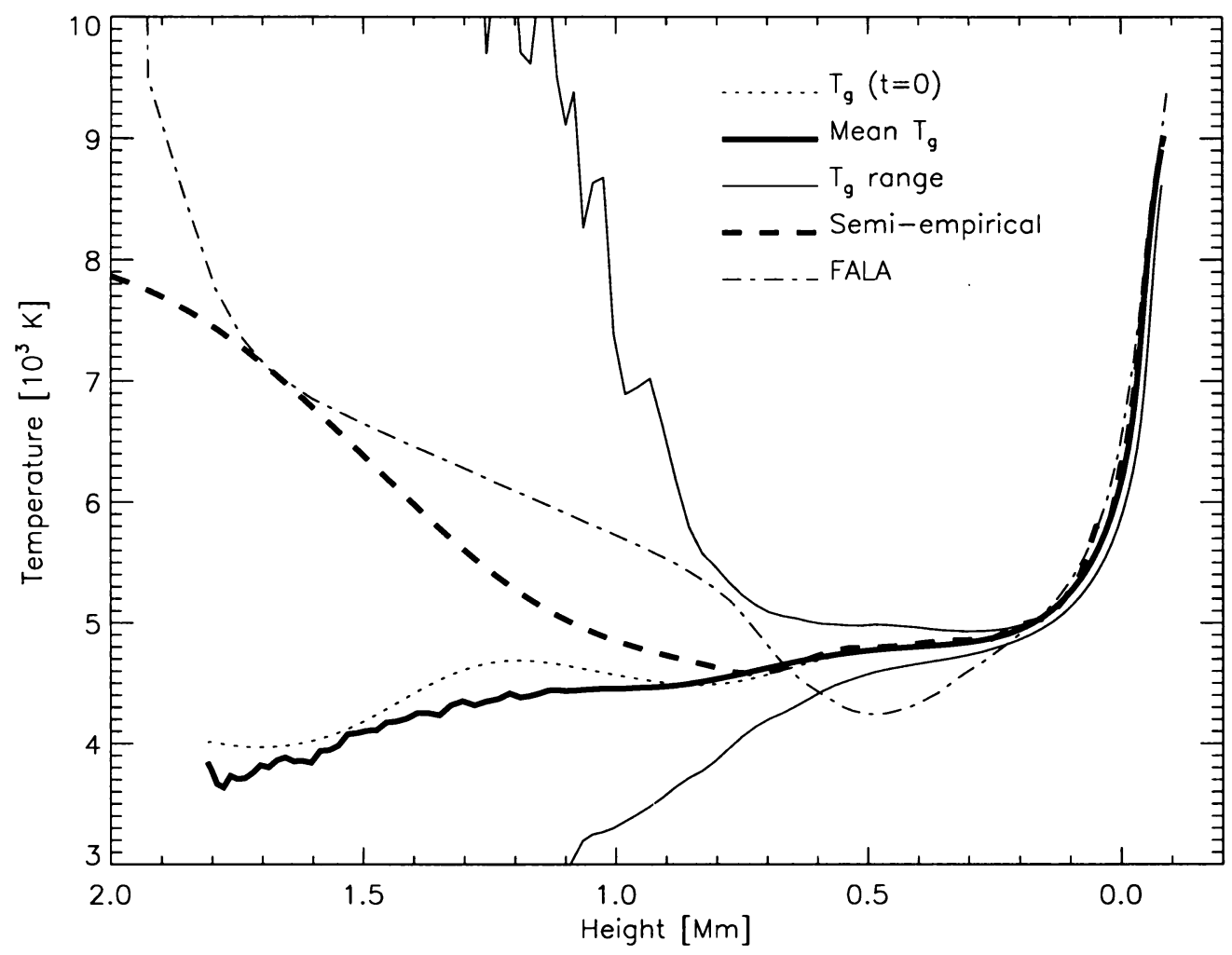

Figure 3. Time average of the temperature in the dynamical simulation, the range of temperatures in the simulation, the semi-empirical model that gives the best fit to the time average of the intensity as a function of wavelength calculated from the dynamical simulation, the starting model for the dynamical simulation and the semi-empirical model FAL-A. The maximum temperatures are only reached in narrow shock spikes of short duration. The semi-empirical model giving the same intensities as the dynamical simulation shows a chromospheric temperature rise while the mean temperature in the simulation does not. From Carlsson \& Stein (1995).

\section{Dynamic Diagnostics}

In a dynamic atmosphere one must be cautious in applying traditional methods used to analyze static atmospheres:

- the height of formation of a spectral feature varies in time. Increased temperature may lead to increased opacity and a formation higher in the atmosphere where the temperature is lower. Increased temperature may thus lead to a decrease in intensity. 
- the Doppler-shift of a spectral line typically has a response height higher than the intensity. This will cause phase differences between Doppler-shift and intensity to be different than the phase differences between fluid velocity and temperature.

- slow rates may cause the ionization/excitation equilibrium to depend on the previous history of the atmosphere and deviate from the equilibrium of the instantaneous atmosphere. This is the case for hydrogen where the ionization/recombination time-scale is longer than the dynamic time-scale in the Solar chromosphere (see Carlsson \& Stein 1992).

- contribution functions may be multi-peaked with one peak close to optical depth unity and another at a temperature spike in a shock (see Carlsson \& Stein 1995).

In the following sections we present two examples of dynamic diagnostics.

\subsection{DETERMINING VELOCITY AND TEMPERATURE FROM LINE OBSERVATIONS}

As pointed out above the Doppler-shift and intensity of a line may be bad proxies for the fluid velocity and temperature. In a dynamic model we have access to all quantities and may test different diagnostic methods. One such method is to use the Doppler-shift and intensity of the line core to diagnose the velocity and temperature at the height of formation. Different spectral lines with different opacities will give information from different heights. Stein \& Carlsson (1996) compared line center Doppler-shifts and temperatures with actual fluid values for several sodium lines concluding that the fluid velocity and line core Doppler-shift are tightly correlated in the photospheric lines but more loosely correlated in the chromospheric D-line. The correlations between line core radiation temperature and fluid temperatures is much looser because of decoupling of the source function from the Planck function.

Observationally it may be difficult to simultaneously observe a large number of spectral lines in order to sample different formation heights. Across one spectral line one has in principle information from all heights between the formation height of the line core and the continuum. The difficulty lies in disentangling the effects of Doppler-shifts and intensity changes. One method that attempts this decoupling is the lambda-meter method. Rigid "rods" of given lengths in wavelength are fitted to an observed line-profile. The mid-point of the rod is taken as the Doppler-shift and the intensity value as a proxy for local temperature. The shorter the length of the rod the higher the formation height. An example with 15 different length rods fitted to the profile of the D-line of neutral sodium is given in Fig. 4. The length of the rods and the choice of the sodium D-line was motivated by the work of Deubner et al. (1996) (see also Deubner 1998, these proceedings). They used this technique to study propagation properties of waves in the Solar upper photosphere and lower chromosphere. An important assumption in their work is that the Doppler-shift of the rod is a good proxy for the fluid velocity at a fixed height in the atmosphere. For the interpretation of their intensity-Doppler-shift phase diagrams the additional assumption is that the rod intensity is a good measure of the fluid temperature.

From Fig. 4 it is evident that the Doppler-shift of the rod is a good proxy for the velocity at the mean response height for the line-wing, which is formed in the deep photosphere where the wave amplitude is small. At line-center the correlation is still quite good but with a large scatter. The intensity, however, is a rather bad proxy for the local temperature even deep down. The radiation temperature is systematically lower than the fluid temperature. At line-center there is almost no correlation with 

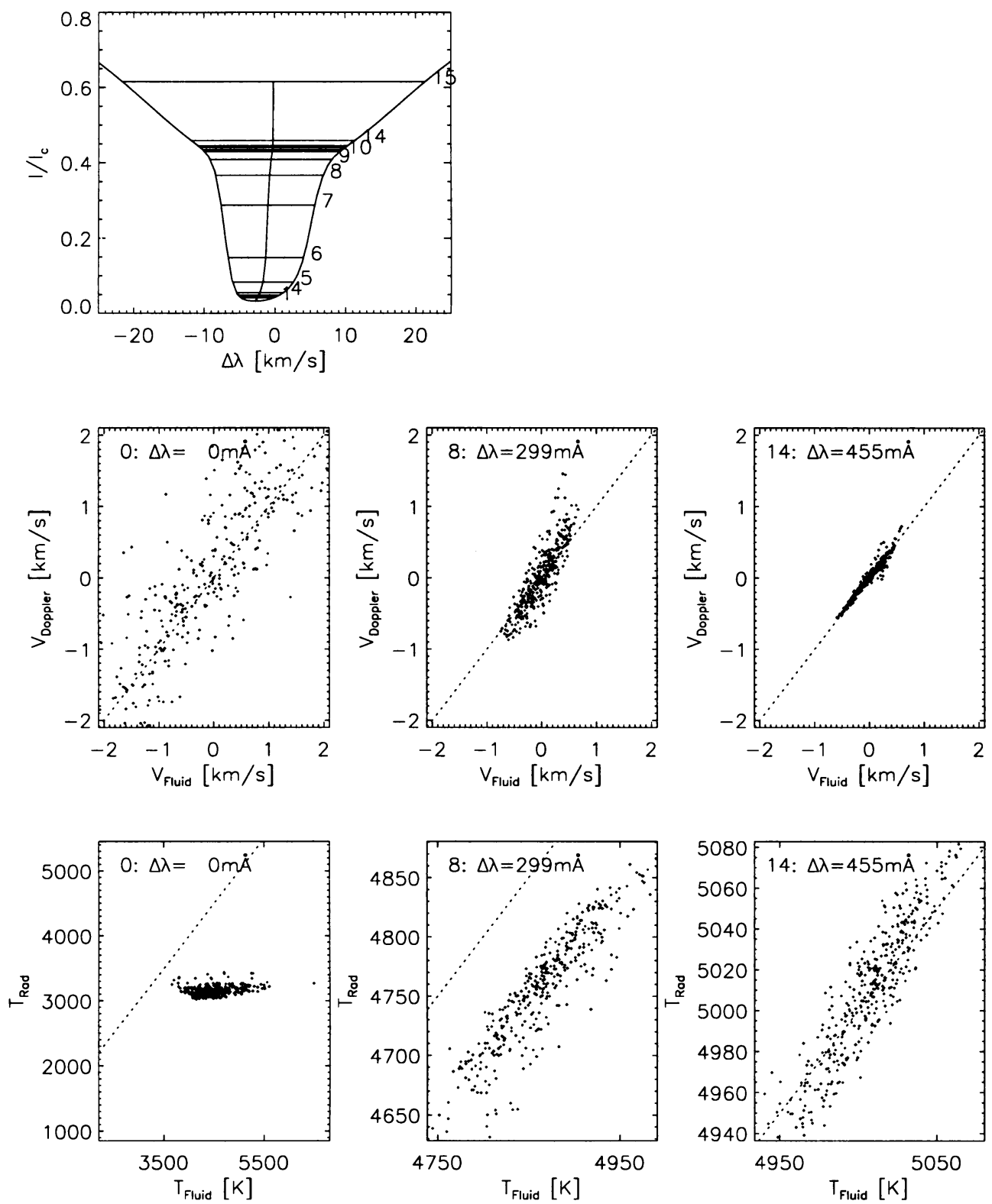

Figure 4. Lambda-meter method. The top panel shows the $\mathrm{NaI}$ line profile from one snapshot in the simulation with the 15 diagnostic rods of given lengths shown. The middle panels show the correlation between the fluid velocity at the static formation height and the Doppler-shift of a given rod (line-center, number 8 and number 14 from left to right). The bottom panels show the correlation between the fluid temperature at these heights and the radiation temperature of the intensity at the position of the rod. The dotted lines show $\mathrm{V}_{\text {Doppler }}=\mathrm{V}_{\text {Fluid }}$ and $\mathrm{T}_{\text {Rad }}=\mathrm{T}_{\text {Fluid }}$. 
the fluid temperature. Doppler-shift - intensity phase relations can thus not be interpreted in terms of a velocity - temperature phase difference. Even Doppler-shift Doppler-shift phase relations have to be treated with caution. Phase diagrams constructed from the measured Doppler-shifts in the simulation show similarities with the phase diagrams of Deubner et al. (1996) with a phase jump close to $7 \mathrm{mHz}$ while phase diagrams constructed from the fluid velocities look more like what is expected from propagating waves. The simulations correspond to only a few spatial points and the phase diagrams are therefore very noisy. For a proper comparison with the observations a much larger simulation would be necessary, preferably in $3 \mathrm{D}$. Such work is in progress.

\subsection{CaII H \& K GRAIN FORMATION}

The $\mathrm{H}$ and $\mathrm{K}$ resonance lines of singly ionized calcium constitute the best available diagnostics of the Solar chromosphere available from the ground. The observations show complex temporal variations with marked line assymetries, see Section 3 . The simulations by Carlsson \& Stein (1997) show how these lines are formed. Since the bottom boundary condition is taken from observed Doppler-shifts of a photospheric line it is possible to compare the computed $\mathrm{H}$-line intensities with the observations. This is done in Fig. 5. The correspondence is remarkable. Slight alterations of the piston velocities give different intensity behaviour. The sensitivity of the results to the driving velocity field together with the good agreement between simulation and observations give confidence that we can extract physics from the simulations that has bearing on the Sun.

The bright grains are produced by shocks near $1 \mathrm{Mm}$ above $\tau_{500}=1$. Shocks in the mid chromosphere produce a large source function (and therefore high emissivity) because the density is high enough for collisions to couple the $\mathrm{Ca}$ II populations to the local conditions. The asymmetry of the line profile is due to velocity gradients near $1 \mathrm{Mm}$. Material motion Doppler-shifts the frequency where atoms emit and absorb photons, so the maximum opacity is located at - and the absorption profile is symmetric about - the local fluid velocity, which is shifted to the blue behind shocks. The optical depth depends on the velocity structure higher up. Shocks propagate generally into downflowing material, so there is little matter above to absorb the blue Dopplershifted radiation. The corresponding red peak is absent because of small opacity at the source function maximum and large optical depth due to overlying material. The brightness of the violet peak depends on the height of shock formation. The lower the shock, the higher the density and the larger the source function. The position in wavelength of the bright violet peak depends on the bulk velocity at the shock peak and the width of the atomic absorption profile (described with the microturbulence fudge parameter).

The bright grains are produced primarily by waves near and slightly above the acoustic cutoff frequency. The precise time and strength of a grain depends on the interference between these waves at the acoustic cutoff frequency and higher frequency waves. When waves near the acoustic cutoff frequency are weak, then higher frequency waves produce grains. The "five-minute" trapped p-mode oscillations are not the source of the grains, although they can modify the behavior of higher frequency waves. The wave pattern that exists at the solar surface is due to the interference of many trapped and propagating modes, so that the grain pattern has a stochastic nature. 

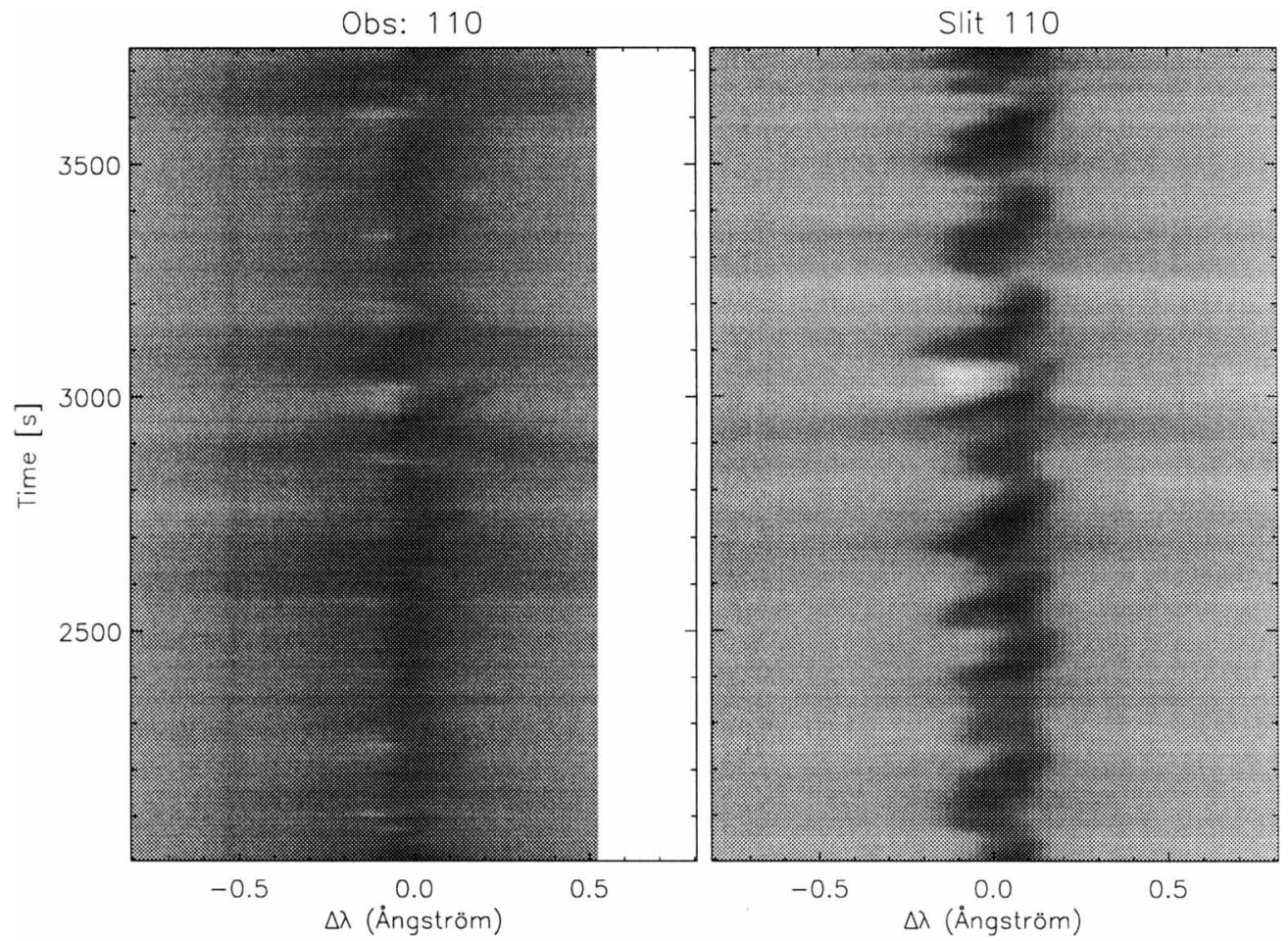

Figure 5. The computed $\mathrm{Ca}$ II $\mathrm{H}$ line intensity as a function of wavelength and time compared with observations.

\section{Summary}

Considerations of basic physics, observations and simulations all end up with the same conclusion: the Solar chromosphere is very dynamic. The very essence of the chromosphere lies in the dynamics. To study the Solar chromosphere with a mean model is as meaningful as taking the mean of Beethoven's 9th symphony (http://www.astro.uio.no/-matsc/iau97/beethoven_mean.au).

\section{References}

Bocchialini, K., Vial, J.-C., Koutchmy, S. 1994, ApJ, 423, L67

Carlsson, M., Judge, P. G., Wilhelm, K. 1997, ApJ, 486, L63

Carlsson, M., Stein, R. F. 1992, ApJ, 397, L59

Carlsson, M., Stein, R. F. 1994, in M. Carlsson (ed.), Proc. Mini-Workshop on Chromospheric Dynamics, Institute of Theoretical Astrophysics, Oslo, p. 47

Carlsson, M., Stein, R. F. 1995, ApJ, 440, L29

Carlsson, M., Stein, R. F. 1997, ApJ, 481, 500

Deubner, F. L., Waldschik, T., Steffens, S. 1996, A\&A, 307, 936

Fontenla, J. M., Avrett, E. H., Loeser, R. 1993, ApJ, 406, 319

Gustafsson, B. 1973, Uppsala Astr. Obs. Ann., 5, No. 6 
Harvey, J., Jefferies, S., Pomerantz, M., Duvall, T., J. 1992, BAAS, 180, 1705

Hofmann, J., Steffens, S., Deubner, F. L. 1996, A\&A, 308, 192

Lites, B. W. 1985, in H. U. Schmidt (ed.), Theoretical Problems in High Resolution Solar Physics, MPA/LPARL Workshop, Max-Planck-Institut für Physik und Astrophysik MPA 212, München, p. 273

Lites, B. W., Rutten, R. J., Kalkofen, W. 1993, ApJ, 414, 345

Maltby, P., Avrett, E. H., Carlsson, M., Kjeldseth-Moe, O., Kurucz, R. L., Loeser, R. 1986, ApJ, 306, 284

Rutten, R. J., Uitenbroek, H. 1991, Solar Phys., 134, 15

Solanki, S. K., Livingston, W., Ayres, T. 1994, Science, 263, 64

Steffens, S., Hofmann, J., Deubner, F. L. 1996, A\&A, 307, 288

Stein, R. F., Carlsson, M. 1996, in J. Christensen-Dalsgaard, F. Pijpers (eds.), Solar Convection and Oscillations and their Relationship, Proc. of AArhus Workshop

Thomas, R. N., Athay, R. G. 1961, Physics of the Solar Chromosphere, Interscience, New York

Uitenbroek, H., R.W.Noyes, Rabin, D. 1994, ApJ, 432, L67

Vernazza, J. E., Avrett, E. H., Loeser, R. 1981, ApJS, 45, 635

Von Uexkuell, M., Kneer, F. 1995, A\&A, 294, 252 